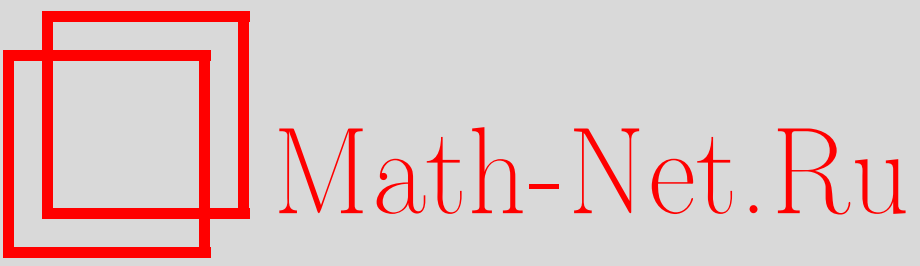

Ю. Г. Прохоров, Замечание о разрешении трехмерных терминальных особенностей, УМН, 2002, том 57, выпуск 4, 187-188

DOI: https://doi.org/10.4213/rm547

Использование Общероссийского математического портала Math-Net.Ru подразумевает, что вы прочитали и согласны с пользовательским соглашением

http://www.mathnet.ru/rus/agreement

Параметры загрузки:

IP: 3.89 .197 .203

26 апреля 2023 г., 12:42:36 


\title{
ЗАМЕЧАНИЕ О РАЗРЕШЕНИИ ТРЕХМЕРНЫХ ТЕРМИНАЛЬНЫХ ОСОБЕННОСТЕЙ
}

\author{
Ю. Г. ПрохоРОВ
}

Пусть $(Z, o)$ - трехмерная терминальная особенность индекса $r \geqslant 1$, пусть $\varphi: \widetilde{Z} \rightarrow Z$-ее разрешение, и пусть $S \subset \widetilde{Z}$ - исключительный дивизор такой, что $\operatorname{Center}(S)=$ o. Ясно, что бирациональньй тип $S$ не зависит от $g$. По $[1 ; 2.14]$ поверхность $S$ бирационально линейчатая. Мы говорим, что соответствующее дискретное нормирование $\nu=\nu_{S}$ поля функции $\mathscr{K}(Z)$ рачионально (соответственно бирационально линейчатое) если такова поверхность $S$. Нас интересует существование рациональных исключительных дивизоров над $o \in Z$ с малшіми дискрепантностями:

Теорема. Пусть $(Z, o)$ - трехмерная терминальная особенность типа $c A / r, r \geqslant 1$, и пусть $\nu$ - дивизориальное дискретное нормирование поля $\mathscr{K}(Z)$ такое, что а $(\nu) \leqslant 1$ $u$ Center $_{Z}(\nu)=$ o. Тогда $\nu$ рачионально.

Напомним, что согласно классификации [2], [3] особенность $(Z, o)$ принадлежит одному из следующих классов: $c A / r, c A x / 4, c A x / 2, c D / 2, c D / 3, c E / 2, c D, c E$. В серии работ [4; Приложение], [5], [6] было доказано, что для любого $i \in \mathbb{N} \backslash r \mathbb{N}$ существует исключительньй дивизор $S$ с центром в $о$ и дискрепантностью $a(S)=i / r$ (см. также [7] и [8]). Отметим, что исключительные дивизоры с дискрепантностями $<1$ появляются в любом разрешении. Дивизоры с дискрепантностью 1 и Center $=$ о появляются в любом дивизориальном разрешении, т.е. в разрешении таком, что исключительное множество имеет чистую коразмерность 1.

ДоКАЗАТЕльСтво теОРемЫ. Пусть $F \in\left|-K_{Z}\right|$ - общий элемент. Тогда $(F, o)$ - дювалевская особенность (типа $\left.A_{n}\right)[3 ; 6.4]$. По обращению присоединения $[9 ; 17.6]$ пара $(Z, F)$ чисто логтерминальна. Пусть $q: Z^{q} \rightarrow Z$ - $\mathbb{Q}$-факториализация (см., например, [9;6.7]). Тогда $Z^{q}$ имеет (терминалшные) особенности типов $c A / r_{i}$. Действительно, поверхность $F^{q}:=q^{-1}(F)$ содержит все особые точки многообразия $Z^{q}$ и $F^{q} \in\left|-K_{Z^{q}}\right|$. Так как $F^{q} \rightarrow F$-крепантный морфизм, то $F^{q}$ может иметь особенности только типов $A_{n_{i}}$. Таким образом, заменяя $Z$ на $Z^{q}$, мы можем считать, что $Z \mathbb{Q}$-фракториально.

Пусть $S$ - исключительный дивизор с центром в $o$ и дискрепантностью $a(S) \leqslant 1$. Тогда $a(S, F)<1$. Так как $K_{Z}+F$ линейно тривиален, то мы имеем $a(S, F) \leqslant 0$. Далее, имеется 1 -дополнение $K_{F}+\Theta$ для $K_{F}$ вблизи $o$ (см. [4; 5.2.3]). Согласно [10;4.4.1] это дополнение может быть продолжено на $Z$, т.е. имеется (целый) дивизор Картье $F^{\prime}$ такой, что $\left.F^{\prime}\right|_{F}=\Theta$, $K_{Z}+F+F^{\prime} \sim 0$ и пара $\left(Z, F+F^{\prime}\right)$ логканонична. Тогда $a\left(S, F+F^{\prime}\right)=-1$. Теперь наша теорема - следствие следуюшего простого факта.

ПредЛОЖениЕ. Пусть $(Z, o)$ - трехмерная $\mathbb{Q}$-факториальная терминальная особенность, и пусть $\nu$ - дивизориальное дискретное нормирование поля $\mathscr{K}(Z)$. Предположим, что существует граница $D$ такая, что пара $(Z, D)$ логканонична и $a(\nu, D)=-1$. Тогда

(i) нормирование $\nu$ рационально или бирационально әквивалентно линейчатой поверхности над эллиптической кривой;

(ii) если, кроме того, $\lfloor D\rfloor$ имеет по крайней мере две компоненты, проходящие через о, то д рачионально.

ДоказАтЕльство. Согласно [9; 17.10] существует раздутие $f: X \rightarrow Z$ с неприводимьм исключительным дивизором $S$, представляющим нормирование $\nu$, такое, что логдивизор $K_{X}+S+$ $D_{X}=f^{*}\left(K_{Z}+D\right)$ логканоничен. Здесь $D_{X}-$ собственньй прообраз $D$. В этой ситуации имеем $\rho(X / Z)=1$, следовательно, $D_{X} \equiv-\left(K_{X}+S\right) f$-обилен.

Рассмотрим минимальную логтерминальную модификацию $g: Y \rightarrow X$ пары $\left(X, S+D_{X}\right)$ (см., например, $[10 ; 3.1 .3])$, т.е. раздутие такое, что $Y \mathbb{Q}$-факториалшно и

$$
K_{Y}+S_{Y}+D_{Y}+E=g^{*}\left(K_{X}+S+D_{X}\right)
$$

Работа выполнена при частичной поддержке грантов INTAS-OPEN 269 и РФФИ 02-01-00441. 
является дивизориально логтерминальным. Здесь $S_{Y}, D_{Y}-$ собственные прообразы $S$ и $D_{X}$ соответственно, а $E=\sum E_{i}$ - (приведенный) исключительный дивизор с $a\left(E_{i}, S+D_{X}\right)=-1$. Обозначим $\Delta:=\operatorname{Diff}_{S_{Y}}\left(D_{Y}+\sum E_{i}\right)$ и $\Omega:=\left.f^{*} D_{X}\right|_{S_{Y}}$. По $[9 ; 17.7]$ поверхность $S_{Y}$ нормальна, а пара $\left(S_{Y}, \Delta\right)$ логканонична. Утверждение (i) легко следует из леммы ниже.

Чтобы доказать (ii), предположим, что поверхность $S_{Y}$ нерациональна и дивизор $\lfloor D\rfloor$ приводим. Тогда $S \cap\left\lfloor D_{X}\right\rfloor$ имеет по крайней мере две неприводимые компоненты. То же самое верно для $S_{Y} \cap\left\lfloor D_{Y}+E\right\rfloor$. По лемме ниже $\lfloor\Delta\rfloor$ имеет в точности две компоненты (содержащиеся в $\left.\left\lfloor D_{Y}+E\right\rfloor\right)$ и пара $\left(S_{Y}, \Delta\right)$ чисто логтерминальна. Далее, $\left(S_{Y}, \Delta-\varepsilon \Omega\right)$ логтерминальна по Кавамате при $0<\varepsilon$. Так как дивизор $\Omega$ численно эффективен и объемен, то для $0<\varepsilon \ll 1$ пара $\left(S_{Y}, \Delta-\varepsilon \Omega\right)$ является логповерхностью дель Пеццо с логтерминальными по Кавамате особенностями. В этой ситуации поверхность $S_{Y}$ рациональна (см., например, [10; 5.4.1]).

Лемма. Пусть $(S, \Delta)$ - проективная логповерхность с $\kappa(S)=-\infty$. Предположим, что дивизор $K_{X}+\Delta$ логканоничен и численно тривиален и что поверхность $S$ нерачиональна. Тогда $S$ - бирачионально линейчатая поверхность над әллиптической кривой и существует не более двух дивизоров с дискрепантностью $a(\cdot, \Delta)=-1$.

ДокаЗАТЕльство (ср. [4;6.9]). Заменим $S$ на минимальное разрешение, а $\Delta$ - на крепантный прообраз. Имеется стягивание $\phi: S \rightarrow C$ (с общим слоем $\left.-\mathbb{P}^{1}\right)$ на кривую $C$ рода $p_{a}(C) \geqslant 1$. В этой ситуации пара $(S, \Delta)$ имеет лишь канонические особенности и все компоненты $\Delta$ горизонтальны $[10 ; 8.2 .2-8.2 .3]$. Следовательно, дивизоры с дискрепантностью $a(\cdot, \Delta)=-1$ - это в точности компоненты $|\Delta|$. Отсюда немедленно получается, что число дивизоров с дискрепантностью -1 не больше двух. Если $\lfloor\Delta\rfloor \neq 0$, то для любой компоненты $\Delta_{i} \subset\lfloor\Delta\rfloor$ имеем $2 p_{a}\left(\Delta_{i}\right)-2 \leqslant$ $\left(K_{S}+\Delta\right) \cdot \Delta_{i}=0$. Поэтому $p_{a}(C) \leqslant p_{a}\left(\Delta_{i}\right) \leqslant 1$. Осталось рассмотреть случай, когда пара $(S, \Delta)$ логтерминальна по Кавамате. Для любой компоненты $\Delta_{i} \subset \operatorname{Supp}(\Delta)$ имеем $\Delta_{i}^{2} \leqslant 0$ (иначе $\left(S, \Delta+\varepsilon \Delta_{i}\right)$ - логповерхность дель Пецио с логтерминальными по Кавамате особенностями). Тогда, как и выше, $p_{a}(C) \leqslant p_{a}\left(\Delta_{i}\right)=\frac{1}{2}\left(K_{S}+\Delta_{i}\right) \cdot \Delta_{i}+1 \leqslant \frac{1}{2}\left(K_{S}+\Delta\right) \cdot \Delta_{i}+1=1$.

Отметим, что утверждение (ii) нашей теоремы неверно для других типов терминалшных особенностей:

ПримеР [4; Приложение]. Пусть $(Z, o)$ - терминальная $c A x / 2$-особенность $\left\{x^{2}+y^{2}+z^{4 m}+\right.$ $\left.t^{4 m}=0\right\} / \mathbb{Z}_{2}(0,1,1,1)$. Рассмотрим взвешенное раздутие с весами $\frac{1}{2}(2 m, 2 m+1,1,1)$. Тогда исключительный дивизор $S$ задается в $\mathbb{P}(2 m, 2 m+1,1,1)$ уравнением $x^{2}+z^{4 m}+t^{4 m}=0$. Он приведен, неприводим и $a(S)=\frac{1}{2}$. Легко видеть, что $S$ - бирационально линейчатая поверхность над гиперэллиптической кривой рода $2 m-1$.

\section{СПИСОК ЛИТЕРАТУРЫ}

[1] M. Reid // Journées de géométrie algébrique, Angers, France / ed. A. Beauville. Rockville: Sijthoff \& Noordhoff, 1980. P. 273-310. [2] S. Mori // Nagoya Math. J. 1985. V. 98. P. 43-66. [3] M. Reid // Proc. Sympos. Pure Math. 1987. V. 46. P. 343-416. [4] В. В. Шокуров // Изв. АН CССР. Сер. матем. 1992. Т. 56. С. 105-203. С приложением Ю. Каваматы. [5] D. Markushevich // J. Math. Sci. Tokyo. 1996. V. 3. № 2. P. 445-456. [6] В. В. Шокуров // Изв. РАН. Cep. матем. 1993. T. 57. № 2. C. 165-222. [7] V. V. Shokurov // J. Math. Sci. 1996. V. 81. № 3. P. 2667-2699. [8] T. Hayakawa // Publ. Res. Inst. Math. Sci. 1999. V. 35. № 3. P. 515-570. [9] J. Kollár (ed.). Flips and Abundance for Algebraic Threefolds. Paris: Soc. Math. France, 1992. (Astérisque. V. 211.) [10] Yu. G. Prokhorov. Lectures on Complements on log Surfaces. Tokyo: Math. Soc. Japan, 2001. (Mem. Math. Soc. Japan. V. 10.)

Московский государственный

Принято редколлегией университет им. М.В. Ломоносова 23.05 .2002

E-mail: prokhoro@mech.math.msu.su 